\title{
Physico-chemical stability of astaxanthin nanodispersions prepared with polysaccharides as stabilizing agents
}

\begin{abstract}
The emulsification and stabilization ability of four selected polysaccharides, namely, gum Arabic, xanthan gum, pectin and methyl cellulose, in the preparation of water-dispersible astaxanthin nanoparticles using the emulsification-evaporation technique was investigated in this study. The chemical and molecular structure of polysaccharides had significant effects $(\mathrm{p}<0.05)$ on the physicochemical properties of the prepared astaxanthin nanodispersions. Among all prepared nanodispersions, sample produced and stabilized using gum Arabic showed the smallest average particle size $(295 \mathrm{~nm})$ and highest physical stability. The observed considerable degradation of astaxanthin in the resulting nanodispersions during processing $(24-70 \% \mathrm{w} / \mathrm{w})$ and storage at $10{ }^{\circ} \mathrm{C}$ for $30 \mathrm{~d}(86-96 \% \mathrm{w} / \mathrm{w})$ illustrated the limited chemical stability of polysaccharide-stabilized nanodispersions.
\end{abstract}

Keyword: Astaxanthin nanodispersions; Emulsification-evaporation technique;

Polysaccharides 